\title{
Caracterização de rizobios noduladores de feijão-fava (Phaseolus lunatus L.) em solos de três estados do nordeste brasileiro
}

Marineide Rodrigues do Amorim, Antonio Victor Cavalcante Rocha Silva, Jadson Emanuel Lopes Antunes, Louise Melo de Souza Oliveira, Ademir Sérgio Ferreira Araújo

Universidade Federal do Piauí - UFPI. E-mail: asfaruai@yahoo.com.br

\section{Resumo}

O objetivo deste estudo foi caracterizar isolados de rizóbios noduladores de feijão-fava oriundos de solos dos estados do Ceará, Piauí e Maranhão. Amostras de solos foram coletadas de áreas produtoras de feijãofava (Ordem: Fabales, Família: Fabaceae, Espécie: Phaseolus lunatus L.) nos três estados. O experimento foi realizado em vasos, contendo solo de cada local, semeados com feijão-fava. A coleta dos nódulos para isolamento e caracterização foi realizada aos 35 dias após a emergência das plantas. 0 total de 75 isolados foram obtidos e submetidos a caracterização morfológica e fisiológica. Morfologicamente, a maioria dos isolados exibiram colônias circulares, convexas, com bordas inteiras e de superfície lisa. Fisiologicamente, todos os isolados apresentaram crescimento rápido e a maioria apresentou reação ácida, habilidade moderada de produzir muco com consistência gomosa e colônias com coloração creme. Os isolados encontrados nestes solos apresentaram características morfológicas e fisiológicas similares àquelas encontradas nos gêneros Rhizobium, Sinorhizobium, Allorhizobium e Mesorhizobium.

Palavras-chave: diversidade morfofisiológica; simbiose; condições edafoclimáticas.

\section{Characterization of lima bean-nodulating rhizobia in soils from three northeastern Brazilian States}

\begin{abstract}
The aim of this study was to characterize lima bean-nodulating rhizobia from soils of northeastern Brazil. Soil samples were collected in regions with production of lima bean in Ceara, Piaui and Maranhao states. The experiment was conducted in pots containing soil from each region, sowed with lima bean. Nodules were sampled for isolation and characterization at 35 days after plant emergence. A total of 75 isolates were found and submitted to morphological and physiological characterization. Morphologically, the majority of isolates exhibited circular and convex colonies with entire border and smooth surface. Physiologically, all isolated presented fast growth, and the majority of isolates presented acid reaction, moderate ability to produce mucus with gummy consistency and colonies with cream coloration. The isolates found in this study presented similar characteristics to genera Rhizobium, Sinorhizobium, Allorhizobium e Mesorhizobium.
\end{abstract}

Keywords: Phaseolus lunatus; leguminous; tropical soils.

\section{Introdução}

O feijão-fava (Phaseolus lunatus L.) é uma das espécies do gênero Phaseolus de grande importância socioeconômica especialmente na região Nordeste do Brasil. A espécie, cultivada principalmente na América Latina, apresenta grande diversidade genética e capacidade de adaptação à diferentes ambientes (ARAÚJO et al.,
2017). Além disto, o feijão-fava tem a capacidade de realizar simbiose com bactérias diazotróficas, comumente conhecidas por rizóbios, responsáveis pelo processo de Fixação Biológica do Nitrogênio (FBN) e, desta forma, fornecer o nutriente às plantas (BARAÚNA et al., 2014). O processo ocorre no interior de nódulos que são formados a partir de sinalizações moleculares 
entre a planta e a bactéria. Além disso, a FBN é influenciada por fatores edafoclimáticos que podem reduzir a eficiência do processo (DABESSA et al., 2018).

Desta forma, o isolamento e caracterização de rizóbios noduladores do feijão-fava em solos de áreas produtoras da cultura pode possibilitar a identificação e futura seleção de estirpes adaptadas as condições edafoclimáticas presentes. No Brasil, a caracterização de isolados noduladores em leguminosas tem sido utilizada como ferramenta na identificaçãoo de bactérias simbiontes em feijão-comum e feijão-caupi (CARDOSO et al., 2017; CHAGAS JUNIOR et al., 2013). Em feijão-fava, estudos utilizaram a caracterização de isolados na identificação de rizóbios noduladores em solos do Peru e México que são considerados centros de diversidade da espécie (ORMEÑO-ORRILLO et al., 2006; LÓPEZLÓPEZ et al., 2013; DURÁN et al., 2014; MOTTAALDANA et al., 2010). Estes estudos identificaram os gêneros Bradyrhizobium e Rhizobium como principais gêneros noduladores de feijão-fava no Peru e no México (ORMEÑO et al., 2007; LOPEZLOPEZ et al., 2013).

Por outro lado, na região nordeste do Brasil, provável centro de domesticação pela grande diversidade genética presente, os estudos são incipientes na caracterização de bactérias noduladoras na espécie. $O$ principal estudo de caracterização de isolados de rizóbios em feijãofava no Nordeste brasileiro foi conduzido por Santos et al. (2011) em solo do Piauí e que, utilizando uma abordagem morfofisiológica, identificaram os gêneros Bradyrhizobium, Rhizobium e Sinorhizobium como simbiontes da espécie. Desta forma, a caracterização de isolados de rizóbios em solos de outras regiões produtoras do Nordeste possibilitaria ampliar o conhecimento sobre a diversidade de bactérias fixadoras de $\mathrm{N}$ noduladoras do feijão-fava e, possivelmente, subsidiaria indicações comerciais para inoculantes. Além disso, as condições edafoclimáticas de cada região influenciam na diversidade dos rizóbios. Neste sentido, a principal hipótese deste estudo é que as diferentes características das regiões produtoras de feijão-fava contribuiriam para ampliar a diversidade de rizóbios noduladores da espécie. Portanto, o objetivo deste estudo foi caracterizar isolados de rizóbios oriundos de solos dos estados do Ceará, Piauí e Maranhão.

\section{Material e Métodos}

O experimento foi conduzido em casa de vegetação do Departamento de Fitotecnia do Centro de Ciências Agrárias da Universidade Federal do Piauí (UFPI) (0505'21" S e 4248'07" W) entre abril e julho de 2017, Teresina/ Piauí. As amostras de solos utilizadas no experimento foram oriundas de regiões produtoras de feijãofava nos municípios de Tianguá - Ceará (03 43' 56" S; 40 59' 30" W), Várzea Grande - Piauí (06응 32' 41" S; 42 14' 47" W) e São Domingos do Maranhão - Maranhão ( $5^{\circ} 34^{\prime} 46^{\prime \prime} \mathrm{S}, 44^{\circ} 22^{\prime}$ 59" W).

O experimento foi conduzido em vasos com capacidade de $5 \mathrm{~kg}$ cada em triplicata. De cada localidade foram coletadas $15 \mathrm{~kg}$ de solo a uma profundidade de $0-20 \mathrm{~cm}$ em 10 pontos distintos. $O$ delineamento experimental utilizado foi o inteiramente casualizado com três repetições. Em cada vaso foram semeadas oito sementes de feijão-fava, genótipo UFPI-491 ("Boca de Moça") oriunda do Banco Ativo de Germoplasma de feijão-fava (BAG de feijão-fava) da UFPI. Após sete dias do plantio, o desbaste foi realizado deixando uma planta por vaso. A correção do solo e a adubação química (80 $\mathrm{kg} \mathrm{ha}^{-1}$ $\mathrm{P}_{2} \mathrm{O}_{5}, 20 \mathrm{~kg}$ ha $^{-1} \mathrm{~K}_{2} \mathrm{O}$ ), sem $\mathrm{N}$, foi realizada de acordo com as recomendações técnicas para a cultura (BEZERRA et al., 2010).

A coleta dos nódulos para isolamento e caracterização foi realizada aos 35 dias após a emergência das plantas. Os nódulos sadios foram lavados em água corrente e armazenados em tubos contendo sílica gel. No isolamento, os nódulos foram reidratados por 12 horas em água destilada autoclavada e, posteriormente, esterilizados com álcool 95\% (30 segundos), hipoclorito de sódio $2 \%$ (2 minutos) (HUNGRIA, 1994). Os três maiores nódulos foram selecionados e macerados em solução salina a $0,85 \%$ de $\mathrm{NaCl}$. Posteriormente, 200 $\mu \mathrm{l}$ do macerado foram colocados em placa de Petri contendo o meio de extrato de levedura-manitolágar-YMA (VINCENT, 1970), em triplicata, com o reagente Vermelho Congo $0,025 \%$ (v/v) (HUNGRIA, 1994). Os isolados foram submetidos a coloração de Gram e aqueles que apresentaram reação negativa, indicativas de rizóbio, foram armazenados em $4^{\circ} \mathrm{C}$ para posterior caracterização morfológica e fisiológica.

A caracterização morfológica foi realizada em meio sólido YMA com o indicador ácidobásico azul de bromotimol (VINCENT, 1970). Os isolados foram caracterizados através dos descritores: 1) Forma da colônia (FC- punctiforme 
(até $1 \mathrm{~mm}$ de diâmetro); circular (com mais de 1 $\mathrm{mm}$ de diâmetro); irregular; filamentosa); 2) Elevação da colônia (EC - plana, lenticular, convexa, pulvinada); 3) Bordas da colônia (BCinteiros, ondulados, lobados, denteada, filamentosa); 4) Superfície da colônia (SC- lisa, rugosa, papilada); 5) Caracteres ópticos da colônia (DO - opaca, translúcida e transparente) (MELLONI et al., 2006).

A caracterização fisiológica foi realizada em meio YMA com indicador azul de bromotimol, considerando os descritores: 1) tempo de crescimento (TC: MR- muito rápido, 1 dia, $R$ rápido, 2 a 3 dias, 1 - intermediário - 4 a 5 dias, Llento- 6 a 15 dias) (MARTINS et al., 1997); 2) formação de ácidos e álcalis (FAA: AC - ácida, Nneutra, AL - alcalina), 3) formação de muco (FM: $A$ - ausente, $P$ - presente); 4) volume do muco (VM: UM - muito, $M$ - médio, $P$ - pouco e $S$ seco); 5) elasticidade do muco (EM: P - presença de fio, A - ausência de fio), 6) Cromogenia ou Pigmentação da colônia - amarela, branca, creme, incolor (MELLONI et al., 2006).
$\mathrm{Na}$ análise de divergência genética entre os isolados bacterianos noduladores de feijão-fava utilizou-se a moda dos dados referentes às características morfológicas e fisiológicas para obter-se a matriz de dissimilaridade, que posteriormente foi submetida ao método hierárquico UPGMA (Unweighted Pair Group Method with Arithmetic Mean). O corte no dendrograma gerado pelo método UPGMA foi realizado no ponto em que se observou a mudança abrupta de nível, conforme recomendado por Cruz et al., (2004). As análises do dendrograma foram realizadas com auxílio do programa GENES (CRUZ, 2013).

\section{Resultados e Discussão}

Um total de 75 isolados foram caracterizados, sendo 17,17 e 41 oriundos dos solos dos estados do Ceará, Piauí e Maranhão, respectivamente. Os resultados da caracterização morfológica e fisiológica estão apresentados nas Tabelas 1, 2 e 3. 
Tabela 1. Características morfológicas e fisiológicas dos isolados de rizóbios noduladores de feijão-fava, oriundos de solo de São Domingos do Maranhão, Maranhão.

\begin{tabular}{|c|c|c|c|c|c|c|c|c|c|c|}
\hline \multirow{2}{*}{ Isolados } & \multicolumn{5}{|c|}{ Características morfológicas ${ }^{1}$} & \multicolumn{5}{|c|}{ Características fisiológicas $^{2}$} \\
\hline & $\mathrm{FC}$ & EC & $\mathrm{BC}$ & $\mathrm{SC}$ & DO & TC & $\mathrm{pH}$ & PM & TM & CC \\
\hline MA01 & $\mathrm{Cl}$ & $\mathrm{CV}$ & IT & LS & $\mathrm{TL}$ & $\mathrm{RP}$ & $A C$ & $A D$ & VC & AM \\
\hline MA03 & $\mathrm{Cl}$ & CV & IT & LS & $\mathrm{TL}$ & $\mathrm{RP}$ & $A C$ & $\mathrm{MD}$ & GM & AM \\
\hline MA06 & $\mathrm{Cl}$ & CV & IT & LS & $\mathrm{OP}$ & $\mathrm{RP}$ & $A C$ & MD & GM & AM \\
\hline MA07 & $\mathrm{Cl}$ & CV & IT & LS & TL & $\mathrm{RP}$ & $A C$ & $A D$ & GM & AM \\
\hline MA08 & $\mathrm{EL}$ & CV & $\mathrm{IT}$ & LS & $\mathrm{TL}$ & $\mathrm{RP}$ & $\mathrm{NE}$ & MD & GM & $\mathrm{CR}$ \\
\hline MA11 & $\mathrm{Cl}$ & $\mathrm{CV}$ & $\mathrm{IT}$ & LS & $\mathrm{OP}$ & $\mathrm{RP}$ & $A C$ & $A D$ & $\mathrm{GM}$ & AM \\
\hline MA12 & $\mathrm{Cl}$ & $\mathrm{PL}$ & $\mathrm{IT}$ & LS & $\mathrm{TL}$ & $\mathrm{RP}$ & $A C$ & $M D$ & $\mathrm{GM}$ & $C R$ \\
\hline MA13 & EL & $\mathrm{CV}$ & $\mathrm{IT}$ & LS & $\mathrm{OP}$ & $\mathrm{RP}$ & $A C$ & $P C$ & GM & $C R$ \\
\hline MA14 & $\mathrm{Cl}$ & $\mathrm{PL}$ & OD & LS & OP & $\mathrm{RP}$ & $A C$ & $P C$ & GM & AM \\
\hline MA15 & IR & $\mathrm{CV}$ & OD & RG & $\mathrm{OP}$ & $\mathrm{RP}$ & $A C$ & $P C$ & GM & AM \\
\hline MA17 & $\mathrm{Cl}$ & $\mathrm{PL}$ & $\mathrm{IT}$ & LS & $\mathrm{TL}$ & $\mathrm{RP}$ & $A C$ & $\mathrm{EC}$ & SC & CR \\
\hline MA18 & $\mathrm{Cl}$ & $\mathrm{CV}$ & $\mathrm{IT}$ & LS & $\mathrm{TL}$ & $\mathrm{RP}$ & $A C$ & $M D$ & VC & $C R$ \\
\hline MA20 & $\mathrm{Cl}$ & PL & $\mathrm{IT}$ & LS & OP & $\mathrm{RP}$ & $A C$ & $P C$ & GM & $C R$ \\
\hline MA21 & $\mathrm{Cl}$ & $\mathrm{CV}$ & $\mathrm{IT}$ & LS & TL & $\mathrm{RP}$ & $A C$ & $M D$ & GM & $C R$ \\
\hline MA22 & $\mathrm{Cl}$ & $\mathrm{PL}$ & $\mathrm{IT}$ & LS & $\mathrm{TL}$ & $\mathrm{RP}$ & $A C$ & $\mathrm{EC}$ & $\mathrm{SC}$ & $C R$ \\
\hline MA25 & $\mathrm{Cl}$ & $\mathrm{CV}$ & $\mathrm{IT}$ & LS & OP & $\mathrm{RP}$ & $A C$ & $P C$ & GM & AM \\
\hline MA27 & $\mathrm{Cl}$ & $\mathrm{CV}$ & $\mathrm{IT}$ & $\mathrm{RG}$ & $\mathrm{OP}$ & $\mathrm{RP}$ & $A C$ & $\mathrm{MD}$ & VC & CR \\
\hline MA28 & $\mathrm{Cl}$ & $\mathrm{CV}$ & $\mathrm{IT}$ & LS & $\mathrm{TL}$ & $\mathrm{RP}$ & $A C$ & $A D$ & VC & $C R$ \\
\hline MA29 & $\mathrm{Cl}$ & $\mathrm{CV}$ & $\mathrm{IT}$ & LS & $\mathrm{TL}$ & $\mathrm{RP}$ & $A C$ & $A D$ & $\mathrm{GM}$ & AM \\
\hline MA31 & $\mathrm{Cl}$ & $\mathrm{CV}$ & $\mathrm{IT}$ & LS & $\mathrm{OP}$ & $\mathrm{RP}$ & $A C$ & $M D$ & GM & CR \\
\hline MA32 & $\mathrm{Cl}$ & $\mathrm{PL}$ & $\mathrm{IT}$ & LS & $\mathrm{OP}$ & $\mathrm{RP}$ & $\mathrm{NE}$ & $\mathrm{EC}$ & SC & $C R$ \\
\hline MA33 & IR & $\mathrm{PL}$ & OD & LS & $O P$ & $\mathrm{RP}$ & $A C$ & $\mathrm{MD}$ & $\mathrm{GM}$ & $C R$ \\
\hline MA34 & IR & $P L$ & $\mathrm{IT}$ & $\mathrm{RG}$ & $\mathrm{OP}$ & $\mathrm{RP}$ & $A C$ & $M D$ & $\mathrm{GM}$ & $C R$ \\
\hline MA36 & $\mathrm{Cl}$ & $\mathrm{CV}$ & $\mathrm{IT}$ & $\mathrm{RG}$ & $\mathrm{OP}$ & $\mathrm{RP}$ & $A C$ & $A D$ & $\mathrm{GM}$ & $C R$ \\
\hline MA37 & $\mathrm{Cl}$ & $\mathrm{PL}$ & $\mathrm{IT}$ & RG & TL & $\mathrm{RP}$ & $A C$ & $M D$ & GM & $C R$ \\
\hline MA39 & $\mathrm{Cl}$ & $\mathrm{CV}$ & $\mathrm{IT}$ & RG & $\mathrm{OP}$ & $\mathrm{RP}$ & $A C$ & $M D$ & VC & $C R$ \\
\hline MA41 & $\mathrm{Cl}$ & PL & $\mathrm{IT}$ & LS & OP & $\mathrm{RP}$ & $A C$ & $P C$ & GM & $C R$ \\
\hline MA42 & IR & $\mathrm{PL}$ & OD & $\mathrm{RG}$ & $\mathrm{TL}$ & $\mathrm{RP}$ & $A C$ & $\mathrm{EC}$ & SC & $C R$ \\
\hline MA45 & $\mathrm{Cl}$ & $\mathrm{PL}$ & $\mathrm{IT}$ & RG & $\mathrm{OP}$ & $\mathrm{RP}$ & $A C$ & $M D$ & GM & CR \\
\hline MA46 & IR & $\mathrm{CV}$ & $\mathrm{IT}$ & LS & $\mathrm{TL}$ & $\mathrm{RP}$ & $A C$ & $M D$ & VC & AM \\
\hline MA48 & $\mathrm{Cl}$ & $\mathrm{CV}$ & OD & LS & $\mathrm{OP}$ & $\mathrm{RP}$ & $A C$ & $M D$ & GM & CR \\
\hline MA49 & $\mathrm{Cl}$ & $\mathrm{PL}$ & $\mathrm{IT}$ & LS & $\mathrm{OP}$ & $\mathrm{RP}$ & $A C$ & $\mathrm{PC}$ & $\mathrm{GM}$ & $C R$ \\
\hline MA51 & $\mathrm{Cl}$ & $\mathrm{CV}$ & $\mathrm{IT}$ & LS & $\mathrm{TL}$ & $\mathrm{RP}$ & $A C$ & $M D$ & GM & $C R$ \\
\hline MA52 & $\mathrm{Cl}$ & $\mathrm{PL}$ & $\mathrm{IT}$ & LS & $\mathrm{TL}$ & $\mathrm{RP}$ & $A C$ & $M D$ & GM & AM \\
\hline MA53 & $\mathrm{Cl}$ & $\mathrm{PL}$ & $O D$ & LS & $\mathrm{TL}$ & $\mathrm{RP}$ & $A C$ & $M D$ & $\mathrm{GM}$ & CR \\
\hline MA54 & $\mathrm{Cl}$ & $\mathrm{CV}$ & OD & LS & $\mathrm{TL}$ & $\mathrm{RP}$ & $A C$ & $A D$ & VC & CR \\
\hline MA55 & $\mathrm{Cl}$ & $\mathrm{CV}$ & $\mathrm{IT}$ & LS & $\mathrm{TL}$ & $\mathrm{RP}$ & $A C$ & $M D$ & $\mathrm{GM}$ & $C R$ \\
\hline MA56 & $\mathrm{IR}$ & $\mathrm{CV}$ & $O D$ & LS & $\mathrm{TL}$ & $\mathrm{RP}$ & $A C$ & $M D$ & GM & AM \\
\hline MA59 & $\mathrm{Cl}$ & $\mathrm{PL}$ & $\mathrm{IT}$ & RG & $\mathrm{OP}$ & $\mathrm{RP}$ & $A C$ & $\mathrm{MD}$ & $\mathrm{GM}$ & AM \\
\hline MA61 & $\mathrm{IR}$ & $\mathrm{CV}$ & $\mathrm{IT}$ & LS & $\mathrm{OP}$ & $\mathrm{RP}$ & $A C$ & $M D$ & $\mathrm{GM}$ & $C R$ \\
\hline MA62 & IR & PL & $\mathrm{IT}$ & LS & $\mathrm{TL}$ & $\mathrm{RP}$ & $A C$ & $\mathrm{MD}$ & GM & $C R$ \\
\hline
\end{tabular}

${ }^{1}$ Morfológicas: FC - forma (Cl: circular, IR: irregular, EL: elipsóide); EC - elevação (CV: convexa, PL: plana);BC - borda (IT: inteira, OD: ondulada);SC- superfície (LS: lisa, RG: rugosa); DO- detalhes ópticos (TL: translúcida, OP: opaco) ${ }^{2}$ Fisiológicas: TC - tempo de crescimento (RP: rápido); pH- formação de ácido e álcalis (AC: ácida, NE: neutro); PMprodução de muco (AD: abundante, MD: moderada; PC: pouco; EC: escassa), TM - consistência do muco (GM: gomosa; VC: viscosa (elástica); SC: seca), CC- cor (CR: creme, AM: amarelo). 
Morfologicamente, a maioria dos isolados exibiram colônias circulares, convexas, com bordas inteiras e de superfície lisa. Entretanto, as colônias opacas foram mais frequentes entre os isolados do solo do Ceará, enquanto nos solos do Piauí e Maranhão predominaram isolados com colônias translúcidas. Fisiologicamente, todos os isolados apresentaram crescimento rápido.
Por outro lado, a maioria dos isolados apresentou reação ácida, habilidade moderada de produzir muco com consistência gomosa e colônias com coloração creme. Entretanto, um total de dez isolados apresentou abundância de muco.

Tabela 2: Características morfológicas e fisiológicas dos isolados de rizóbios noduladores de feijão-fava, oriundos de solo de Tianguá, Ceará.

\begin{tabular}{|c|c|c|c|c|c|c|c|c|c|c|}
\hline \multirow{2}{*}{ Isolados } & \multicolumn{5}{|c|}{ Características morfológicas ${ }^{1}$} & \multicolumn{5}{|c|}{ Características fisiológicas ${ }^{2}$} \\
\hline & $\mathrm{FC}$ & $\mathrm{EC}$ & $\mathrm{BC}$ & SC & DO & TC & $\mathrm{pH}$ & PM & TM & $\mathrm{CC}$ \\
\hline CE02 & $\mathrm{Cl}$ & $\mathrm{CV}$ & IT & LS & OP & $\mathrm{RP}$ & $A C$ & MD & GM & CR \\
\hline CEO3 & $\mathrm{Cl}$ & $\mathrm{CV}$ & OD & LS & OP & $\mathrm{RP}$ & $\mathrm{NE}$ & PC & VC & CR \\
\hline CE06 & $\mathrm{Cl}$ & $\mathrm{CV}$ & OD & LS & $\mathrm{TL}$ & $\mathrm{RP}$ & $\mathrm{AC}$ & $M D$ & GM & AM \\
\hline CE10 & EL & $\mathrm{CV}$ & $\mathrm{IT}$ & LS & OP & $\mathrm{RP}$ & $A C$ & $M D$ & GM & CR \\
\hline CE15 & $\mathrm{Cl}$ & $\mathrm{CV}$ & IT & LS & $\mathrm{TL}$ & $\mathrm{RP}$ & $A C$ & MD & $\mathrm{GM}$ & $\mathrm{CR}$ \\
\hline CE17 & $\mathrm{IR}$ & $\mathrm{CV}$ & OD & LS & $\mathrm{OP}$ & $\mathrm{RP}$ & $\mathrm{AC}$ & $M D$ & GM & CR \\
\hline CE18 & $\mathrm{Cl}$ & $\mathrm{CV}$ & $\mathrm{IT}$ & LS & $\mathrm{OP}$ & $\mathrm{RP}$ & NE & $M D$ & GM & CR \\
\hline CE19 & $\mathrm{Cl}$ & $\mathrm{CV}$ & $\mathrm{IT}$ & LS & $\mathrm{TL}$ & $\mathrm{RP}$ & $\mathrm{NE}$ & $\mathrm{MD}$ & $\mathrm{GM}$ & $\mathrm{CR}$ \\
\hline CE27 & $\mathrm{EL}$ & $\mathrm{PL}$ & $\mathrm{IT}$ & LS & $\mathrm{TL}$ & $\mathrm{RP}$ & $A C$ & $P C$ & GM & CR \\
\hline CE32 & $\mathrm{Cl}$ & $\mathrm{CV}$ & $\mathrm{IT}$ & LS & $\mathrm{TL}$ & $\mathrm{RP}$ & $A C$ & $M D$ & VC & CR \\
\hline CE34 & $\mathrm{Cl}$ & $\mathrm{CV}$ & $\mathrm{IT}$ & LS & OP & $\mathrm{RP}$ & $A C$ & $A D$ & GM & AM \\
\hline CE36 & $\mathrm{Cl}$ & $\mathrm{CV}$ & $\mathrm{IT}$ & LS & $\mathrm{OP}$ & $\mathrm{RP}$ & $\mathrm{NE}$ & $M D$ & GM & CR \\
\hline CE40 & $\mathrm{Cl}$ & $\mathrm{CV}$ & $\mathrm{IT}$ & LS & $\mathrm{TL}$ & $\mathrm{RP}$ & $A C$ & $M D$ & GM & CR \\
\hline CE43 & $\mathrm{Cl}$ & $\mathrm{PL}$ & $\mathrm{IT}$ & $\mathrm{RG}$ & OP & $\mathrm{RP}$ & $A C$ & EC & VC & IN \\
\hline CE48 & $\mathrm{Cl}$ & $\mathrm{CV}$ & $\mathrm{IT}$ & $\mathrm{RG}$ & $\mathrm{OP}$ & $\mathrm{RP}$ & $A C$ & $P C$ & GM & AM \\
\hline CE49 & $\mathrm{Cl}$ & $\mathrm{CV}$ & OD & LS & $\mathrm{TL}$ & $\mathrm{RP}$ & $\mathrm{NE}$ & $M D$ & $\mathrm{GM}$ & $\mathrm{CR}$ \\
\hline CE52 & $\mathrm{Cl}$ & $\mathrm{CV}$ & $\mathrm{IT}$ & LS & $\mathrm{TL}$ & $\mathrm{RP}$ & $\mathrm{NE}$ & $\mathrm{MD}$ & $\mathrm{GM}$ & CR \\
\hline
\end{tabular}

${ }^{1}$ Morfológicas: FC - forma (Cl: circular, IR: irregular, EL: elipsóide); EC - elevação (CV: convexa, PL: plana);BC - borda (IT: inteira, OD: ondulada);SC- superfície (LS: lisa, RG: rugosa); DO- detalhes ópticos (TL: translúcida, OP: opaco) ${ }^{2}$ Fisiológicas: TC - tempo de crescimento (RP: rápido); $\mathrm{pH}$ - formação de ácido e álcalis (AC: ácida, NE: neutro); PMprodução de muco (AD: abundante, MD: moderada; PC: pouco; EC: escassa), TM - consistência do muco (GM: gomosa; VC: viscosa (elástica); SC: seca), CC- cor (CR: creme, AM: amarelo). 
Tabela 3: Características morfológicas e fisiológicas dos isolados de rizóbios noduladores de feijão-fava, oriundos de solo de Várzea Grande, Piauí.

\begin{tabular}{|c|c|c|c|c|c|c|c|c|c|c|}
\hline \multirow{2}{*}{ Isolados } & \multicolumn{5}{|c|}{ Características morfológicas ${ }^{1}$} & \multicolumn{5}{|c|}{ Características fisiológicas ${ }^{2}$} \\
\hline & FC & EC & $\mathrm{BC}$ & SC & DO & TC & $\mathrm{pH}$ & PM & TM & CC \\
\hline $\mathrm{PIO2}$ & $\overline{E L}$ & CV & OD & LS & $\overline{T L}$ & $\mathrm{RP}$ & $A C$ & MD & GM & CR \\
\hline $\mathrm{PI03}$ & $\mathrm{Cl}$ & CV & $\mathrm{IT}$ & RG & OP & $\mathrm{RP}$ & $A C$ & PC & GM & CR \\
\hline $\mathrm{PIO4}$ & $\mathrm{Cl}$ & $C V$ & OD & LS & $\mathrm{TL}$ & $\mathrm{RP}$ & $A C$ & $\mathrm{MD}$ & $\mathrm{GM}$ & $C R$ \\
\hline PI08 & $\mathrm{Cl}$ & CV & $\mathrm{IT}$ & LS & $\mathrm{TL}$ & $\mathrm{RP}$ & $A C$ & MD & VC & CR \\
\hline PI09 & $\mathrm{IR}$ & $\mathrm{PL}$ & OD & RG & OP & $\mathrm{RP}$ & $A C$ & $\mathrm{MD}$ & $\mathrm{GM}$ & AM \\
\hline PI10 & $\mathrm{Cl}$ & $\mathrm{PL}$ & $\mathrm{IT}$ & LS & $\mathrm{TL}$ & $\mathrm{RP}$ & $A C$ & MD & GM & CR \\
\hline $\mathrm{PI} 11$ & $\mathrm{Cl}$ & CV & $\mathrm{IT}$ & LS & $\mathrm{TL}$ & $\mathrm{RP}$ & $A C$ & $A D$ & $\mathrm{GM}$ & AM \\
\hline PI12 & $\mathrm{Cl}$ & CV & $\mathrm{IT}$ & $\mathrm{RG}$ & $\mathrm{TL}$ & $\mathrm{RP}$ & $A C$ & $P C$ & $\mathrm{GM}$ & CR \\
\hline PI13 & EL & CV & OD & LS & OP & RP & $A C$ & PC & VC & $C R$ \\
\hline PI17 & $\mathrm{IR}$ & $C V$ & $\mathrm{IT}$ & LS & $\mathrm{TL}$ & $\mathrm{RP}$ & $A C$ & $\mathrm{MD}$ & $\mathrm{GM}$ & CR \\
\hline PI18 & $\mathrm{Cl}$ & $\mathrm{CV}$ & IT & LS & $\mathrm{TL}$ & $\mathrm{RP}$ & $A C$ & $\mathrm{MD}$ & $\mathrm{GM}$ & $\mathrm{CR}$ \\
\hline PI21 & EL & CV & $\mathrm{IT}$ & LS & $\mathrm{TL}$ & $\mathrm{RP}$ & $A C$ & $A D$ & VC & AM \\
\hline $\mathrm{PI} 25$ & IR & PL & OD & LS & $\mathrm{TL}$ & $\mathrm{RP}$ & $A C$ & $\mathrm{MD}$ & VC & $\mathrm{CR}$ \\
\hline PI26 & $\mathbb{I R}$ & CV & $\mathrm{IT}$ & LS & $\mathrm{TP}$ & $\mathrm{RP}$ & NE & EC & SC & IN \\
\hline PI40 & $\mathrm{Cl}$ & $\mathrm{PL}$ & $\mathrm{IT}$ & $\mathrm{RG}$ & OP & $\mathrm{RP}$ & $A C$ & PC & $\mathrm{GM}$ & $\mathrm{CR}$ \\
\hline PI45 & $\mathrm{Cl}$ & CV & $\mathrm{IT}$ & LS & $\mathrm{TL}$ & $\mathrm{RP}$ & $A C$ & $\mathrm{MD}$ & $\mathrm{GM}$ & AM \\
\hline PI49 & $\mathrm{Cl}$ & PL & OD & LS & OP & $\mathrm{RP}$ & NE & PC & $\mathrm{GM}$ & $\mathrm{CR}$ \\
\hline
\end{tabular}

${ }^{1}$ Morfológicas: FC - forma (Cl: circular, IR: irregular, EL: elipsóide); EC - elevação (CV: convexa, PL: plana);BC - borda (IT: inteira, OD: ondulada);SC- superfície (LS: lisa, RG: rugosa); DO- detalhes ópticos (TL: translúcida, OP: opaco) ${ }^{2}$ Fisiológicas: TC - tempo de crescimento (RP: rápido); $\mathrm{pH}$ - formação de ácido e álcalis (AC: ácida, NE: neutro); PMprodução de muco (AD: abundante, MD: moderada; PC: pouco; EC: escassa), TM - consistência do muco (GM: gomosa; VC: viscosa (elástica); SC: seca), CC- cor (CR: creme, AM: amarelo).

Estes resultados indicam uma diversidade entre os rizóbios noduladores do feijão-fava nos três estados e sugere que as diferenças nas condições edafoclimáticas e localização geográfica exercem influência sobre os tipos e a distribuição de comunidades microbianas na rizosfera.

As características fisiológicas de acidificação do meio são indicativas de bactérias que podem apresentar adaptação a condições ácidas de solo. Esta característica é importante uma vez que o $\mathrm{pH}$ do solo é um fator limitante a FBN, influenciando na sobrevivência da bactéria e na nodulação (RUFINI et al., 2011). Desta forma, a presença de isolados com adaptação a acidez pode ser uma vantagem para a seleção de estirpes. Em relação à produção de muco apresentada pela maioria dos isolados, os resultados indicam habilidades dos isolados na sobrevivência a condições ambientais adversas, tais como tolerância a seca e isto é importante, principalmente, para os solos do Nordeste que apresentam períodos de escassez de umidades e altas temperaturas (ALMEIDA et al., 2018; RODRIGUES et al., 2018). Estas características positivas podem ser associadas à habilidade de crescimento rápido apresentado pelo total de isolados. Segundo Santos et al. (2011), o crescimento rápido favorece uma maior sobrevivência no solo, pois os isolados podem estar mais adaptados as condições edafoclimáticas da região. Dentre os gêneros que apresentaram estas características fisiológicas neste estudo estão Rhizobium (HUNGRIA et al., 2016), Sinorhizobium (MATSUBARA; ZÚÑIGADÁVILA, 2015), Allorhizobium, e Mesorhizobium (MARCHETTI et al., 2017). Estes gêneros bacterianos são simbiontes de algumas importantes leguminosas, tais como feijãocomum e feijão-caupi (MELO; ZILLI, 2009; Silva et al., 2009). Esses resultados contrastam com estudos anteriores que reportaram Bradyrhizobium como o principal gênero nodulador do feijão-fava (ORMEÑO-ORRILLO et al., 2006; LÓPEZ-LÓPEZ et al., 2013). Além disso, estas características fisiológicas apresentadas pelos isolados neste estudo corroboram com os resultados apresentados por Silva et al. (2007) na caracterização de rizóbios do solo da região semiárida de Pernambuco. 
Os dendrogramas de dissimilaridade das características morfológicas e fisiológicas mostraram diferentes níveis de agrupamento dos isolados. No solo do Ceará, utilizando-se uma linha de corte de $52 \%$ de dissimilaridade, houve a formação de seis grupos de diversidade (A, B, C, $D, E$ e F). $O$ grupo $B$ reuniu o maior número de isolados, enquantoque os grupos $\mathrm{C}, \mathrm{E}$ e $\mathrm{F}$ foram formados por único isolado (Figura 1).

Figura 2. Dendrograma obtido pelo agrupamento hierárquico UPGMA a partir da matriz de dissimilaridade genética usando caracterização morfofisiológica e bioquímica de isolados rizobianos dos solos do Ceará.

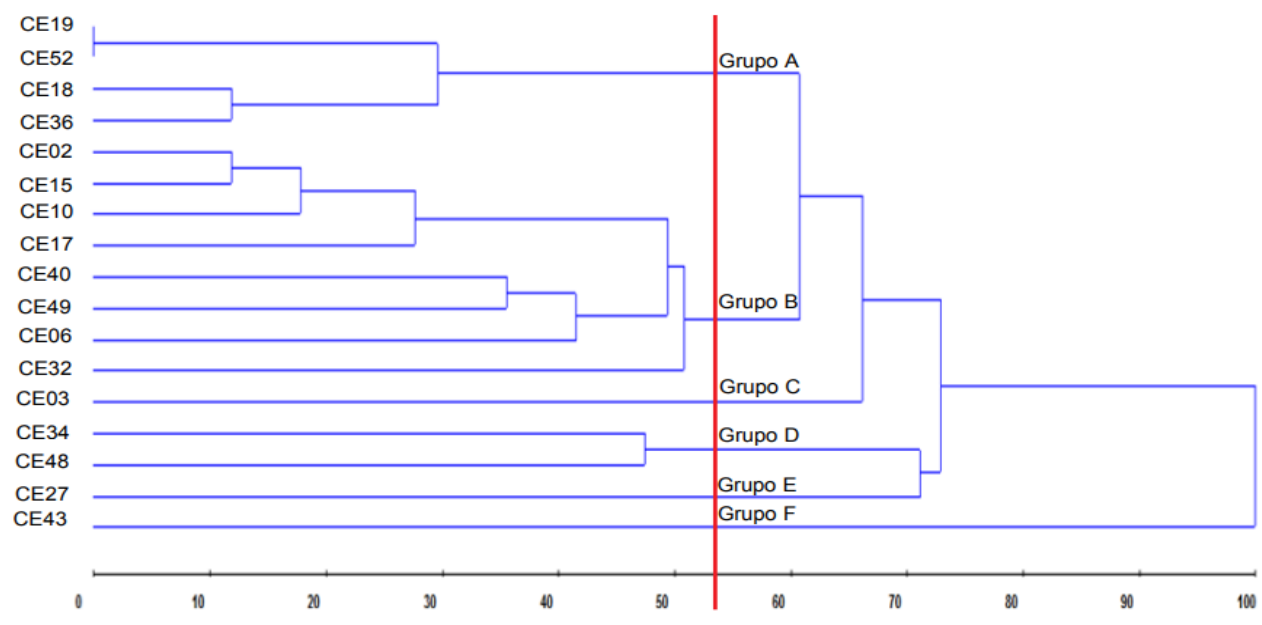

O isolado CE43, pertencente ao grupo $\mathrm{F}$, foi o isolado com o maior número de caracteres fenotípicos divergentes, tais como baixa produções de muco com consistência viscosa e incolor, e, desta forma, agrupou-se aos demais isolados somente a $100 \%$ de dissimilaridade.

No solo do Piauí, a partir de $48 \%$ de dissimilaridade, houve a formação de cinco grupos de diversidade (A, B, C, D e E; Figura 3). Os dados mostraram a formação de dois grupos únicos: D (isolado PI09) e E (isolado PI26). O isolado PI26 agrupou-se aos demais isolados apenas a $100 \%$ de dissimilaridade. Por outro lado, os isolados oriundos do solo do Maranhão formaram 13 grandes grupos de diversidade $(A$, B, C, D, E, F, G, H, I J, K, L e M) (Figura 4).

$O$ grupo $A$ exibiu maior complexidade com 23 isolados, enquanto os grupos B, C, D, E, H, I, K, L, M foram caracterizados por grupos únicos (Figura 4).

Figura 3. Dendrograma obtido pelo agrupamento hierárquico UPGMA a partir da matriz de dissimilaridade genética usando caracterização morfofisiológica e bioquímica de isolados rizobianos dos solos do Piauí.

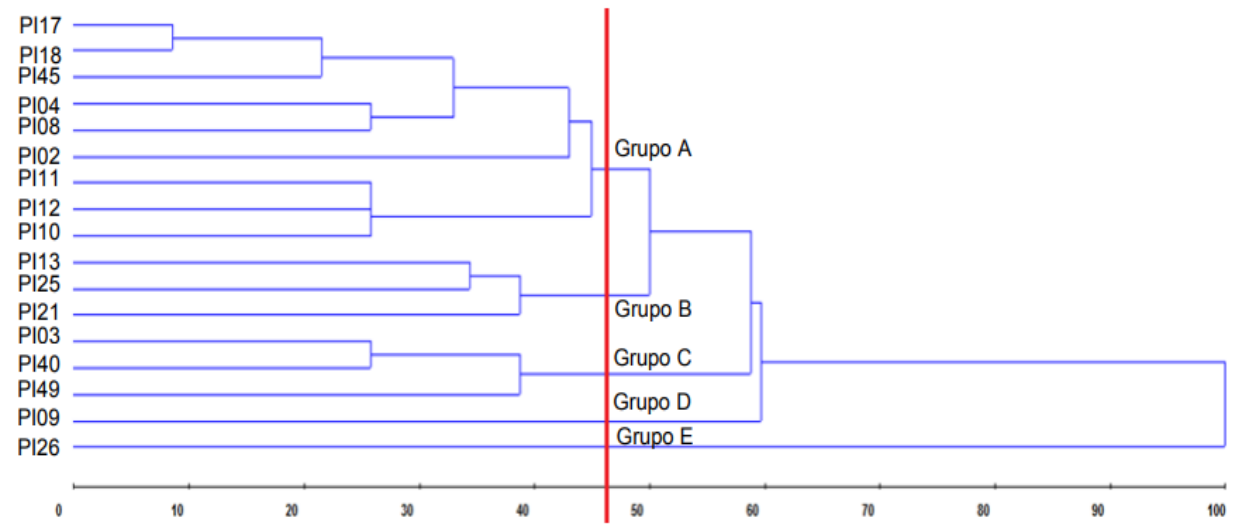


Figura 4. Dendrograma obtido pelo agrupamento hierárquico UPGMA a partir da matriz de dissimilaridade genética usando caracterização morfofisiológica e bioquímica de isolados rizobianos dos solos do Maranhão.

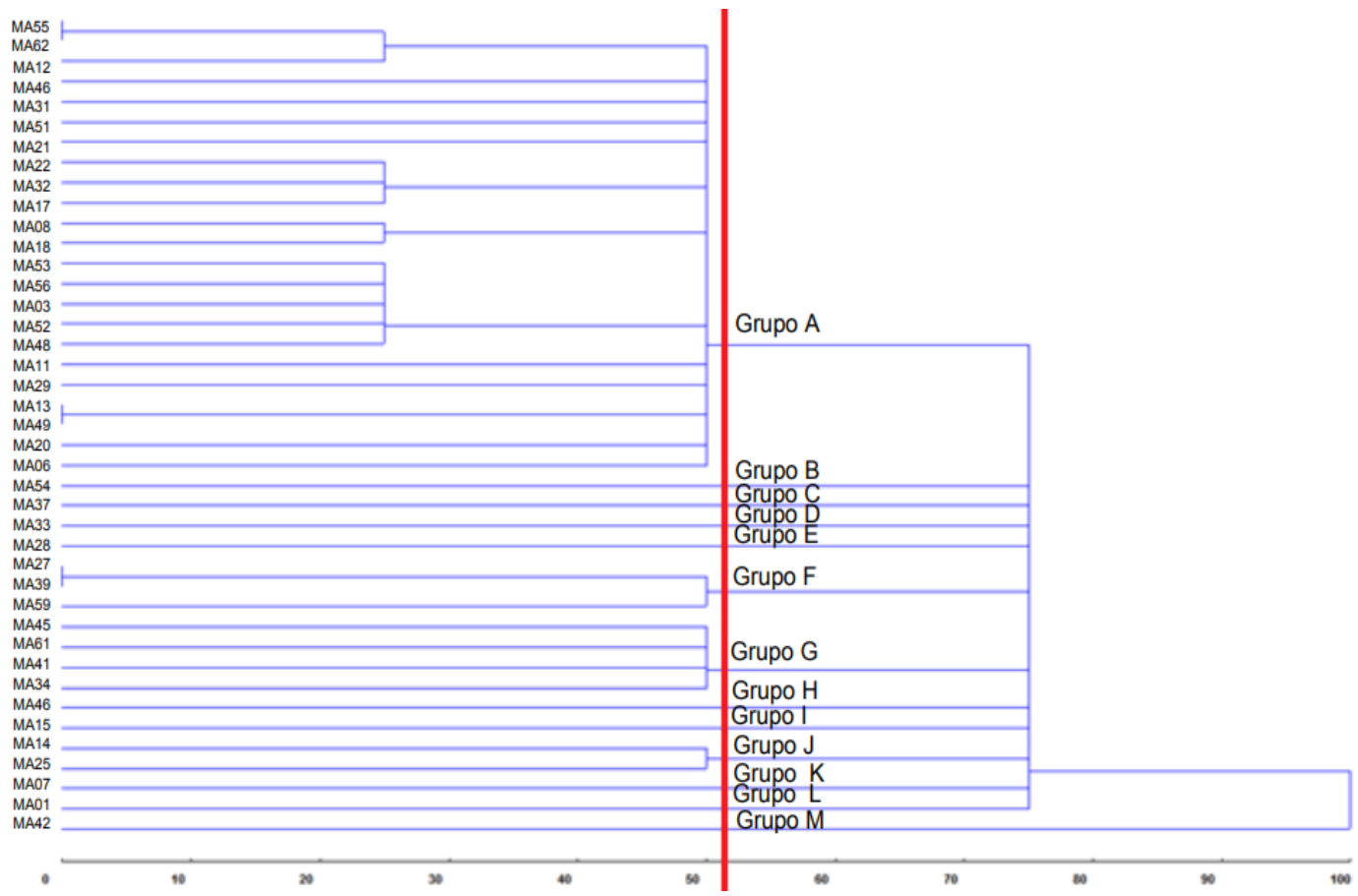

Estes resultados mostram que há uma grande diversidade de rizóbios nativos noduladores de feijão-fava no estado do Nordeste e confirmam os resultados observados em estudos anteriores (SILVA et al., 2007; SANTOS et al., 2011). Por um lado, o comportamento apresentado pelo feijão-fava em relação às bactérias noduladores sugere que a espécie apresenta baixa especificidade hospedeira conforme reportado por Andrews e Andrews (2017) que observaram o feijão-fava sendo nodulado por Bradyrhizobium e Rhizobium. Por outro lado, esta baixa especificidade hospedeira pode indicar a espécie como uma opção entre planta-isca de rizóbios.

\section{Conclusões}

Os solos dos estados do Ceará, Piauí e Maranhão apresentam uma grande diversidade de rizóbios noduladores de feijão-fava. Os isolados encontrados nestes solos apresentaram características morfológicas e fisiológicas similares àquelas encontradas nos gêneros Rhizobium, Sinorhizobium, Allorhizobium e Mesorhizobium. Estudos futuros sobre caracterização molecular e a eficiência destes isolados na FBN em feijão-fava serão importantes para a busca de estirpes para inoculação na cultura.

\section{Referências}

ALMEIDA, A. M. M.; MENDES FILHO, P. F.; GARCIA, K. G. V.; GOMES, V. F. F.; ALMEIDA, C. L. Densidade, caracterização e eficiência de bactérias fixadoras de Nitrogênio em áreas de caatinga degradada. Revista Verde de Agroecologia e Desenvolvimento Sustentável, v. 13, n. 1, p. 16-21, 2018.

https://doi.org/10.18378/rvads.v13i1.5282

ANDREWS, M.; ANDREWS, M. E. Specificity in legume-rhizobia symbioses. International Journal of Molecular Sciences, v. 18, n. 4, p. 705, 2017. https://doi.org/10.3390/ijms18040705

ARAÚJO, A. S. F.; LOPES, Â. C. A.; TERAN, J. C. B. Y.; PALKOVIC, A.; GEPTS, P. Nodulation ability in different genotypes of Phaseolus lunatus by rhizobia from California agricultural soils. Symbiosis, v. 73, n. 1 p. 7-14, 2017. https://doi.org/10.1007/s13199-016-0465-0

BARAÚNA, A. C., SILVA, K., PEREIRA, G. M. D., KAMINSKI, P. E., PERIN, L., ZILLI, J. E. Diversity and nitrogen fixation efficiency of rhizobia isolated from nodules of Centrolobium paraense. Pesquisa Agropecuária Brasileira, v. 49, n. 4, p. 296-305, 2014. 
https://doi.org/10.1590/S0100-

204X2014000400008

BEZERRA, B. R.; ARAÚJO, A. S. F.; SANTOS, J. A.; CARNEIRO, R. F. V. Fertilidade do solo e adubação. In: ARAÚJO, A. S. F.; LOPES, Â. C. A.; GOMES, R. L. F. (Org.). A cultura do feijão-fava na região Meio-Norte do Brasil. 1. ed. Teresina: EDUFPI, 2010. p. 129-138. v. 1.

CARDOSO, A. A., ANDRAUS, M. P., BORBA, T. C. O., MARTIN-DIDONET, C. C. G., FERREIRA, E. P. B. Characterization of rhizobia isolates obtained from nodules of wild genotypes of common bean. Brazilian Journal of Microbiology, v. 48, n. 1, p. 43-50, 2017.

https://doi.org/10.1016/j.bjm.2016.09.002

CHAGAS JÚNIOR, A. F.; OLIVEIRA, L. A.; CASTRO, H. G.; CORNÉLIO, G. L.; SANTOS, G. R.; CHAGAS, L. F. B.; COSTA, J. L. Isolation and phenotypic characterization of rhizobia that nodulate cowpea in the Cerrado in Tocantins State, Brazil. Journal of Biotechnology and Biodiversity, v. 4, n. 3, p. 249-259, 2013. Disponível em: https://repositorio.inpa.gov.br/bitstream/123/47 87/1/isolation.pdf. Acesso em: 21 mar. 2019. https://doi.org/10.20873/jbb.uft.cemaf.v4n3.cha gasjunior

CRUZ, C. D.; REGAZZI, A. J.; CARNEIRO, P. C. S. Modelos biométricos aplicados ao melhoramento genético. Viçosa: UFV, 2004. 480 p. Disponível em: http://www.sidalc.net/cgibin/wxis.exe/?IsisScript=agrono.xis\&method=pos t\&formato $=2 \&$ cantidad $=1$ \& expresion $=\mathrm{mfn}=0054$ 38. Acesso em: 21 mar. 2019.

CRUZ, C. D. GENES - a software package for analysis in experimental statistics and quantitative genetics. Acta Scientiarum, v. 35, n. 3, p. 271-276, 2013. https://doi.org/10.4025/actasciagron.v35i3.2125 $\underline{1}$

DABESSA, A.; ABEBE, Z.; BEKELE, S. Limitations and strategies to enhance biological nitrogen fixation in sub-humid tropics of Western Ethiopia. Journal of Agricultural Biotechnology and Sustainable Development, v. 10, n. 7, p. 122131, 2018.

https://doi.org/10.5897/JABSD2018.0318
DURÁN, D., REY, L., MAYO, J., ZÚÑIGA-DÁVILA, D., IMPERIAL, J., RUIZ-ARGÜESO, T., MARTI'NEZROMERO, E., ORMEÑO-ORRILLO, E. Bradyrhizobium paxllaeri sp. nov. and Bradyrhizobium icense sp. nov., nitrogen-fixing rhizobial symbionts of Lima bean (Phaseolus lunatus L.) in Peru. International journal of systematic and evolutionary microbiology, v. 64, n. 6, p. 2072-2078, 2014.

https://doi.org/10.1099/ijs.0.060426-0

HUNGRIA, M. Coleta de nódulos e isolamento de rizóbio. In: HUNGRIA, M.; ARAUJO, R. S. (Eds.). Manual de métodos empregados em estudo de microbiologia agrícola. Brasília: Embrapa-spi, 1994. p. 45-61.

HUNGRIA, M.; O'HARA, G.; ZILLI, J., ARAUJO, R.; DEAKER, R.; HOWIESON, J. Isolation and growth of rhizobia. In: HOWIESON, J. G.; DILWORTH, M. J. (Eds.). Working with rhizobia. Canberra: Australian Centre for International Agricultural Research (ACIAR), 2016. p. 39-60. Disponível em: http://researchrepository.murdoch.edu.au/id/ep rint/34579. Acesso em: 21 mar. 2019.

LÓPEZ-LÓPEZ, A.; NEGRETE-YANKELEVICH， S.; ROGEL, M. A.; ORMEÑO-ORRILLO, E.; MARTÍNEZ, J.; MARTÍNEZ-ROMERO, E. Native bradyrhizobia from Los Tuxtlas in Mexico are symbionts of Phaseolus lunatus (Lima bean). Systematic and applied microbiology, v. 36, n. 1, p. 33-38, 2013. https://doi.org/10.1016/j.syapm.2012.10.006

MARCHETTI, M. M.; SANTOS, J. C. P.; BARATTO, C. M. Caracterização de bactérias em nódulos de leguminosas arbóreas de fragmentos da floresta ombrófila mista. Scientia agraria, v. 18, n. 4, p. 50-62, 2017. Disponível em:

https://dialnet.unirioja.es/servlet/articulo?codigo =6294990. Acesso em: 21 mar. 2019.

https://doi.org/10.5380/rsa.v18i4.51383

MARTINS, L. M. V.; XAVIER, G. R.; NEVES, M. C. P.; RUMJANEK, N. G. Características relativas ao crescimento em meio de cultura e a morfologia de colônias de "Rizóbio". Seropédica: Embrapa CNPAB, 1997. 14 p. (Série Embrapa CNPAB. Comunicado técnico, 19). Disponível em: https://www.infoteca.cnptia.embrapa.br/bitstrea m/doc/623573/1/cot019.pdf. Acesso em: 21 mar. 2019. 
MATSUBARA, M.; ZÚÑIGA-DÁVILA, D. Phenotypic and molecular differences among rhizobia that nodulate Phaseolus lunatus in the Supe valley in Peru. Annals of microbiology, v. 65, n. 3, p. 18031808, 2015. https://doi.org/10.1007/s13213-015$\underline{1054-9}$

MELO, S. R.; ZILLI, J. E. Fixação biológico de nitrogênio em cultivares de feijão-caupi recomendadas para 0 estado de Roraima. Pesquisa Agropecuária Brasileira, v. 44, n. 9, p. 1177-1183, 2009. https://doi.org/10.1590/S0100$\underline{204 \times 2009000900016}$

MELLONI, R.; MOREIRA, F. M. D. S.; NÓBREGA, R. S. M.; SIQUEIRA, J. O. Eficiência e diversidade fenotípica de bactérias diazotróficas que nodulam caupi [Vigna unguiculata (L.) Walp] e feijoeiro (Phaseolus vulgaris L.) em solos de mineração de bauxita em reabilitação. Revista Brasileira de Ciência do Solo, v.30, n.2, p. 235246, 2006. Disponivel em: https://www.redalyc.org/html/1802/1802140510 05/. Acesso em: 21 mar. 2019.

https://doi.org/10.1590/S0100-

$\underline{06832006000200005}$

MOTTA-ALDANA, J. R., SERRANO-SERRANO, M. L., HERNÁNDEZ-TORRES, J., CASTILLO-VILLAMIZAR, G., DEBOUCK, D. G. Multiple origins of Lima bean landraces in the Americas: evidence from chloroplast and nuclear DNA polymorphisms. Crop Science, v. 50, n. 5, p. 17731787, 2010.

https://doi.org/10.2135/cropsci2009.12.0706

ORMEÑO, E.; TORRES, R.; MAYO, J.; RIVAS, R.; PEIX, A.; VELÁZQUEZ, E.; ZÚNIGA, D. Phaseolus lunatus is nodulated by a phosphate solubilizing strain of Sinorhizobium meliloti in a Peruvian soil. VALÁZQUEZ, E.; RODRÍGUEZ-BARRUECO, C. In: First international meeting on microbial phosphate solubilization. Dordrecht: Springer, 2007. p. 143-147. Disponível em:

https://link.springer.com/chapter/10.1007/9781-4020-5765-6 21. Acesso em: 21 mar. 2019. https://doi.org/10.1007/978-1-4020-5765-6 21

ORMEÑO-ORRILLO, E.; VINUESA, P.; ZUNIGADAVILA, D.; MARTÍNEZ-ROMERO, E. Molecular diversity of native bradyrhizobia isolated from Lima bean (Phaseolus lunatus L.) in Peru. Systematic and applied microbiology, v.
29, n. 3, p. 253-262, 2006.

ttps://doi.org/10.1016/i.syapm.2005.09.002

RODRIGUES, D. R.; SILVA, A. F.; CAVALCANTI, M. I. P.; ESCOBAR, I. E. C.; FRAIZ, A. C. R.; RIBEIRO, P. R. A.; FERREIRA NETO, R. A.; FREITAS, A. D. S.; FERNANDES-JÚNIOR, P. I. Phenotypic, genetic and symbiotic characterization of Erythrina velutina rhizobia from Caatinga dry forest. Brazilian Journal of Microbiology, v. 49, n. 3, p. 503-512, 2018. https://doi.org/10.1016/j.bjm.2017.09.007

RUFINI, M.; FERREIRA, P. A. A.; SOARES, B. L.; OLIVEIRA, D. P.; ANDRADE, M. J. B.; MOREIRA, F. M. S. Simbiose de bactérias fixadoras de nitrogênio com feijoeiro-comum em diferentes valores de $\mathrm{pH}$. Pesquisa Agropecuária Brasileira. v. 46, n.1, p.81-88, 2011. Disponível em: http://seer.sct.embrapa.br/index.php/pab/article Lview/9429/6186. Acesso em: 21 mar. 2019. https://doi.org/10.1590/S0100$\underline{204 X 2011000100011}$

SANTOS, J. O.; ANTUNES, J. E. L.; ARAÚJO, A. S. F.; LYRA, M. C. C. P.; GOMES, R. L. F.; LOPES, A. C. A.; FIGUEIREDO, M. V. B. Genetic diversity among native isolates of rhizobia from Phaseolus lunatus. Annals of Microbiology, v. 61, n. 3, p. 437-444, 2011. https://doi.org/10.1007/s13213$\underline{010-0156-7}$

SILVA, V. N.; SILVA, L. E. D. S. F.; FIGUEIREDO, M. D. V. B.; CARVALHO, F. G.; SILVA, M. L. R. B.; SILVA, A. J. N. Caracterização e seleção de populações nativas de rizóbios de solo da região semi-árida de Pernambuco. Pesquisa Agropecuária Tropical, v.37, n.1, p.16-21, 2007. Disponível em:

https://www.redalyc.org/html/2530/2530202790 04/. Acesso em: 21 mar. 2019.

SILVA, E. F.; MARCHETTI, M. E.; SOUZA, L. C. F.; MERCANTE, F. M.; RODRIGUES, E. T.; VITORINO, A. C. T. Inoculação do feijoeiro com Rhizobium tropici associada à exsudato de Mimosa flocculosa com diferentes doses de nitrogênio. Bragantia, v. 68, n. 2, p. 443-451, 2009. https://doi.org/10.1590/S0006-

$\underline{87052009000200019}$

VINCENT, J. M. A manual for the pratical study of root-nodule-bacteria. Oxford: Blackwells Scientific Publications, 1970. 164 p. 